\title{
Robust Nonlinear Sequential Loop Closure Control Design for an Air-breathing Hypersonic Vehicle Model
}

\author{
Lisa Fiorentini* \\ Andrea Serrani \\ The Ohio State University \\ Columbus, $\mathrm{OH} 43210$, USA
}

\author{
Michael A. Bolender \\ David B. Doman \\ U.S. Air Force Research Laboratory \\ Wright-Patterson AFB, $\mathrm{OH} 45433$, USA
}

\begin{abstract}
This paper describes the design of a nonlinear robust/adaptive controller for an air-breathing hypersonic vehicle model. Due to its complexity, a high fidelity model of the vehicle dynamics derived from first principles is used only in simulations, while a simplified model is adopted for control design. This control-oriented model retains most of the features of the high fidelity model, including non-minimum phase characteristic of the flight-path angle dynamics and strong couplings between the engine and flight dynamics, whereas flexibility effects are regarded as a dynamic perturbation. A nonlinear sequential loop-closure approach is adopted to design a dynamic state-feedback controller that provides stable tracking of velocity and altitude reference trajectories and allows to impose a desired trim value for the angle of attack. Simulation results show that the proposed methodology achieves excellent tracking performances in spite of parameter uncertainties.
\end{abstract}

\section{INTRODUCTION}

Hypersonic air-breathing vehicles offer a promising and cost-effective technology to meet the US Air Force needs for access to space and prompt global strike capabilities. The design of guidance and control systems for hypersonic vehicles presents a set of unique challenges which stem from the peculiar characteristics of the dynamics. In [1] a nonlinear variable structure control approach has been employed, while several other nonlinear control approaches have been proposed for non-flexible models of more general types of vehicles [2]-[5]. A nonlinear controller is designed in [5] applying feedback linearization techniques to a controloriented model derived for the longitudinal dynamical model developed in [6]. Although the simulation results show the effectiveness of this approach, the complexity of the model inevitably leads to very complicated expressions for the high-order Lie derivatives of the regulated outputs and for the controller and thus a robustness analysis cannot be performed.

In this paper we present a general nonlinear design based on both adaptive and robust techniques. As a result the controller does not depend explicitly on the model coefficients and can in principle be designed to provide robustness with respect to parameter uncertainties and variations within a given range. As in [7], the vehicle model considered in this

Work supported by the AFRL/AFOSR Collaborative Center of Control Science at the Ohio State University (Contract No. F33615-01-2-3154.

${ }^{*}$ Corresponding author, Department of Electrical and Computer Engineering, The Ohio State University, 2015 Neil Ave, Columbus, OH. Email: fiorentldece.osu.edu paper is the assumed-modes version [8] of the model of the vehicle longitudinal dynamics developed by Bolender and Doman [6]. Because of the complexity of this model, a control-oriented model has been developed following the approach used in [5]. The outputs to be controlled are the vehicle velocity and altitude. The control inputs are the elevator and canard deflections, and the fuel-to air-ratio of the scramjet engine. The presence of the flexible dynamics is not taken into account directly at the design level, but is regarded as a perturbation on a nominal model, and its effects are evaluated in closed-loop simulations. A classic sequential loop closure approach [9] has been applied by decomposing the vehicle dynamics into controlled subsystems. With respect to our previous work [7], several improvements have been achieved here: the altitude tracking error dynamic equation is included in the stability analysis; the canard control input is endowed by an adaptive mechanism and it is used not only to suppress the non-minimum phase behavior of the system, but also to generate the feed-forward term that creates the desired equilibrium at trim. As a consequence, it is now possible to impose a desired trim value for the angle of attack. The analysis of the flight path angle tracking error dynamics is conducted by resorting to sector boundedness arguments instead of using Taylor series expansions. A simplified stability analysis of the overall system is provided by resorting to Lyapunov stability arguments without using complicated modifications as the ones adopted in [7]. Finally the simulations are performed on the high fidelity model instead of on the simplified curve-fitted model.

The paper is organized as follows: in Section II the control-oriented models is presented. Section III covers the derivation of the controller and simulation results are given in Section IV. Finally, Section V offers a brief summary of the results and a discussion of further work.

\section{VEHICLE MODEL}

The vehicle model considered in this paper (HFM) is the assumed-modes version [8] of [6]. Due its complexity, the HFM is used primarily for simulation. For the development of the controller, a control-oriented model (COM) has been derived by replacing the complex expressions of the aerodynamic forces and moments with curve-fitted approximations [5], and by neglecting the flexible dynamics. 
TABLE I

STATES, CONTROL INPUTS, AND CONTROLLED OUTPUTS

\begin{tabular}{llc} 
States & & \\
\hline$V$ & Vehicle velocity & $V \in[7500,11000] \mathrm{ft} / \mathrm{s}$ \\
$h$ & Altitude & $h \in[85000,135000] \mathrm{ft}$ \\
$\gamma$ & Flight path angle, & \\
$\alpha$ & Angle of attack & $\alpha \in[-0.2,0.2] \mathrm{rad}$ \\
$Q$ & Pitch rate & \multicolumn{1}{c}{$\Phi \in[0,1.5]$} \\
Inputs & & \\
\hline$\Phi$ & Fuel-to-air ratio \\
$\delta_{e}$ & Control surface deflection (elevator) \\
$\delta_{c}$ & Control surface deflection (canard)
\end{tabular}

The COM comprises five rigid-body state variables $x=$ $[V, h, \gamma, \alpha, Q]$ and three control inputs $u=\left[\Phi, \delta_{e}, \delta_{c}\right]$, whereas the output to be controlled is selected as $y=[V, h]$. The equations of motion for the COM are then given by

$$
\begin{aligned}
\dot{V} & =\frac{T \cos \alpha-D}{m}-g \sin \gamma \\
\dot{h} & =V \sin \gamma \\
\dot{\gamma} & =\frac{L+T \sin \alpha}{m V}-\frac{g}{V} \cos \gamma \\
\dot{\alpha} & =-\frac{L+T \sin \alpha}{m V}+Q+\frac{g}{V} \cos \gamma \\
\dot{Q} & =\frac{M}{I_{y y}}
\end{aligned}
$$

where $m$ is the vehicle mass, $I_{y y}$ the moment of inertia and $g$ the acceleration due to gravity. Approximated expressions of the thrust $T$, the pitching moment $M$ about the body $y$-axis, the lift $L$ and drag $D$ are given as

$$
\begin{aligned}
& L \approx \bar{q} S C_{L}\left(\alpha, \delta_{e}, \delta_{c}\right), \quad M \approx z_{T} T+\bar{q} \bar{c} S C_{M}\left(\alpha, \delta_{e}, \delta_{c}\right) \\
& T \approx C_{T, \Phi}(\alpha) \Phi+\bar{C}_{T}(\alpha), \quad D \approx \bar{q} S C_{D}\left(\alpha, \delta_{e}, \delta_{c}\right)
\end{aligned}
$$

where the thrust-to-moment coupling coefficient $z_{T}$, the reference area $S$ and the mean aerodynamic chord $\bar{c}$ are given constants, $\bar{q}$ denotes dynamic pressure and

$$
\begin{aligned}
C_{T, \Phi}(\alpha)= & C_{T}^{\Phi \alpha^{3}} \alpha^{3}+C_{T}^{\Phi \alpha^{2}} \alpha^{2}+C_{T}^{\Phi \alpha} \alpha+C_{T}^{\Phi} \\
\bar{C}_{T}(\alpha)= & \bar{C}_{T}^{3} \alpha^{3}+\bar{C}_{T}^{2} \alpha^{2}+\bar{C}_{T}^{1} \alpha+\bar{C}_{T}^{0} \\
C_{M}\left(\alpha, \delta_{e}, \delta_{c}\right)= & C_{M}^{\alpha^{2}} \alpha^{2}+C_{M}^{\alpha} \alpha+C_{M}^{\delta_{e}} \delta_{e}+C_{M}^{\delta_{c}} \delta_{c}+C_{M}^{0} \\
C_{L}\left(\alpha, \delta_{e}, \delta_{c}\right)= & C_{L}^{\alpha} \alpha+C_{L}^{\delta_{e}} \delta_{e}+C_{L}^{\delta^{\prime}} \delta_{c}+C_{L}^{0} \\
C_{D}\left(\alpha, \delta_{e}, \delta_{c}\right)= & C_{D}^{\alpha^{2}} \alpha^{2}+C_{D}^{\alpha} \alpha+C_{D}^{\delta_{e}^{2}} \delta_{e}^{2}+C_{D}^{\delta_{e}} \delta_{e} \\
& +C_{D}^{\delta_{c}^{2}} \delta_{c}^{2}+C_{D}^{\delta_{c}} \delta_{c}+C_{D}^{0} .
\end{aligned}
$$

\section{Robust/Adaptive Controller Design}

The control problem considered in this paper is to track given velocity and altitude references, $V_{\text {ref }}(t)$ and $h_{\text {ref }}(t)$, generated using filtered step signals. From the altitude reference, a desired trajectory for the flight path angle $\gamma_{\mathrm{d}}(t)$ is derived in such a way that if the flight path angle trajectory follows $\gamma_{\mathrm{d}}(t)$, the altitude tracking error is asymptotically regulated to zero. The initial condition of the vehicle, which will be denoted by $x_{0}=\left[V_{0}, h_{0}, \alpha_{0}, \gamma_{0}, Q_{0}\right]^{\prime}$, is not necessarily a trim condition for the aircraft. For this study, a feasible range for the initial condition is given as

$$
\begin{array}{rlrl}
\left|V_{0}-V_{\text {ref }}(0)\right| & \leq 20 \mathrm{ft} / \mathrm{s} & & \left|h_{0}-h_{\mathrm{ref}}(0)\right| \leq 50 \mathrm{ft} \\
\left|\gamma_{0}-\gamma_{\mathrm{d}}(0)\right| \leq 1.3 \mathrm{deg} & & \left|\alpha_{0}\right| \leq 5 \mathrm{deg}
\end{array}
$$

For the remainder of the work, it is assumed that $x_{0} \in \Xi_{0}$ where $\Xi_{0} \subset \mathbb{R}^{5}$ is a set of initial conditions that satisfies (4). Since the COM is obtained from a curve-fitted approximation of a first-principle model, it is fundamental that the control law provides robustness with respect to uncertainty on the plant model parameters. The vector of all uncertain parameters of the COM will be denoted by $\mathcal{P} \in \mathbb{R}^{p}$. All these parameters are uncertain but assumed to be constant, except for the coefficients $C_{T}^{(\cdot)}$ (which depend on the flight conditions $(h, V)$ ) and the mass of the vehicle $m$, which changes with time due to fuel consumption. We set $m=$ $\tilde{m}(t) \cdot m_{0}$, where $m_{0}$ is the nominal mass value and $\tilde{m}(t)$ is a multiplicative uncertainty. Since $\tilde{m}(t)$ changes slowly with respect to the time scale of the references to be tracked, it is considered constant during each setpoint tracking maneuver. The nominal value of all the uncertain parameters will be denoted by the vector $\mathcal{P}^{0}$ and $\mathcal{P}_{i}^{0}$ will represent the $i$ th component of $\mathcal{P}^{0}$. It will be assumed that $\mathcal{P} \in \Xi_{\mathcal{P}}$ where $\Xi_{\mathcal{P}}$ represents the admissible range of variation of $\mathcal{P}$ and is such that $\mathcal{P}^{0} \subset \Xi_{\mathcal{P}} . \mathbb{P}_{\mathcal{P}_{i}}\left(\Xi_{\mathcal{P}}\right)$ represents the admissible range for the $i$ th element of $\mathcal{P}$.

In this design, we will consider two compact sets, one for the initial condition of the tracking error $e=[\tilde{V}, \tilde{h}, \tilde{\gamma}, \tilde{\alpha}]^{\prime}$, $\Xi_{0}^{e} \subset \mathbb{R}^{4}$ that is compatible with the conditions (4), and another set $\Xi_{\mathrm{wc}}^{e} \supset \Xi_{0}^{e}$ that defines the worst admissible case that we allow for each element of $e$. In particular $\Xi_{\mathrm{wc}}^{e}$ is defined by the condition $e \in \Xi_{\mathrm{wc}}^{e}$ iff:

$$
\begin{array}{ll}
\left|V-V_{\text {ref }}\right| \leq 50 \mathrm{ft} / \mathrm{s} & \left|h-h_{\mathrm{ref}}\right| \leq 120 \mathrm{ft} \\
\left|\gamma-\gamma_{\mathrm{d}}\right| \leq 9.17 \mathrm{deg} & \left|\alpha-\alpha^{*}\right| \leq 2 \mathrm{deg}
\end{array}
$$

The control design aims at finding a Lyapunov function $V(e)$ such that, for a positive value $c$, the level set $\Omega_{c}=\{e$ : $V(e) \leq c\}$ satisfies the following conditions

$\mathcal{C} .1 \quad \Xi_{0}^{e} \subset \Omega_{c} \subset \Xi_{\mathrm{wc}}^{e}$

C.2 the Lie derivative of $V$ along the trajectories of the closed-loop system is negative definite for any $e \in \Omega_{c}$.

These two requirements guarantee that for all initial conditions that satisfy the restrictions (4), the tracking errors remain bounded inside $\Xi_{\mathrm{wc}}^{e}$ and eventually converge to zero. The first step will be to design the controller letting $e$ take values in $\Xi_{\mathrm{wc}}^{e}$, thus, each time we have to bound destabilizing terms or disturbances we will use conditions (5). Then it will be shown that, for all initial conditions that satisfy the restrictions (4), the tracking error closedloop trajectories evolve in the invariant level set $\Omega_{c}$, hence the bounds derived for destabilizing terms or disturbances remain valid for any $t \geq 0$.

\section{A. Adaptive Controller for the Velocity Subsystem}

Substituting the expression of the thrust $T$ in (2) into the first equation of (1), the vehicle velocity dynamics read as $m \dot{V}=C_{T, \Phi}(\alpha) \cos \alpha \Phi+\bar{C}_{T}(\alpha) \cos \alpha-D-m g \sin \gamma$. 
Leting $\tilde{V}:=V-V_{\text {ref }}$, the dynamics of the tracking error for the vehicle velocity is written as

$m \dot{\tilde{V}}=C_{T, \Phi}(\alpha) \cos \alpha \Phi+\bar{C}_{T}(\alpha) \cos \alpha-D-m g \sin \gamma-m \dot{V}_{\text {ref }}$.

By introducing the vector of uncertain parameters $\theta_{1}$, the regressor $\Psi_{1}$ and the input matrix $B_{1}$ respectively as

$$
\begin{gathered}
\theta_{1}=\left[C_{T}^{\Phi \alpha^{3}}, C_{T}^{\Phi \alpha^{2}}, C_{T}^{\Phi \alpha}, C_{T}^{\Phi}, \bar{C}_{T}^{3}, \bar{C}_{T}^{2}, \bar{C}_{T}^{1}, \bar{C}_{T}^{0},\right. \\
\left.S C_{D}^{\alpha^{2}}, S C_{D}^{\alpha}, S C_{D}^{\delta_{e}^{2}}, S C_{D}^{\delta_{e}}, S C_{D}^{\delta_{c}^{2}}, S C_{D}^{\delta_{c}}, S C_{D}^{0}, \tilde{m}\right]^{\prime} \\
\Psi_{1}(x, u)=\left[0,0,0,0,-\alpha^{3} \cos \alpha,-\alpha^{2} \cos \alpha,-\alpha \cos \alpha,\right. \\
\left.-\cos \alpha, \bar{q} \alpha^{2}, \bar{q} \alpha, \bar{q} \delta_{e}^{2}, \bar{q} \delta_{e}, \bar{q} \delta_{c}^{2}, \bar{q} \delta_{c}, \bar{q}, m_{0} g \sin \gamma+m_{0} \dot{V}_{\mathrm{ref}}\right]^{\prime} \\
B_{1}(\alpha)=\left[\alpha^{3} \cos \alpha, \alpha^{2} \cos \alpha, \alpha \cos \alpha, \cos \alpha, 0,0,0,0,\right. \\
0,0,0,0,0,0,0,0]^{\prime},
\end{gathered}
$$

equation (6) can be written in the linearly parameterized form

$$
m \dot{\tilde{V}}=\theta_{1}^{\prime} B_{1}(\alpha) \Phi-\Psi_{1}^{\prime}(x, u) \theta_{1}
$$

Functional controllability of the vehicle [5] implies that $\theta_{1}^{\prime} B_{1}(\alpha) \neq 0$ for all values of $\alpha$ within the feasible envelope of flight conditions and for all possible values assumed by $\theta_{1}$, when each parameter $\mathcal{P}_{i}$ in $\theta_{1}$ varies inside the set $\mathbb{P}_{\mathcal{P}_{i}}\left(\Xi_{\mathcal{P}}\right)$.

Let $\hat{\theta}_{1}$ be a vector of estimates of $\theta_{1}$ and $\tilde{\theta}_{1}:=\hat{\theta}_{1}-$ $\theta_{1}$; let us consider the control Lyapunov function candidate $W_{1}\left(\tilde{V}, \tilde{\theta}_{1}\right)=\frac{m}{2} \tilde{V}^{2}+\frac{1}{2} \tilde{\theta}_{1}^{\prime} \Gamma_{1}^{-1} \tilde{\theta}_{1}$ where $\Gamma_{1}$ is a symmetric positive definite matrix.

Proposition 3.1: Choosing the fuel-to-air ratio input as

$$
\Phi=\frac{1}{\hat{\theta}_{1}^{\prime} B_{1}(\alpha)}\left[-k_{1} \tilde{V}+\Psi_{1}^{\prime}(x, u) \hat{\theta}_{1}\right]
$$

where $k_{1}>0$ is a gain parameter, and the update law for the parameter estimate $\hat{\theta}_{1}$ as

$$
\dot{\hat{\theta}}_{1}=\tilde{V} \Gamma_{1}\left[B_{1}(\alpha) \Phi-\Psi_{1}(x, u)\right],
$$

the Lie derivative of $W_{1}\left(\tilde{V}, \tilde{\theta}_{1}\right)$ along the trajectories of the system is rendered negative semidefinite.

Proof. The Lie derivative of $W_{1}\left(\tilde{V}, \tilde{\theta}_{1}\right)$ along the trajectories of the system is given by

$$
\begin{aligned}
\dot{W}_{1}\left(\tilde{V}, \tilde{\theta}_{1}\right) & =m \tilde{V} \dot{\tilde{V}}+\tilde{\theta}_{1}^{\prime} \Gamma_{1}^{-1} \dot{\hat{\theta}}_{1} \\
& =-k_{1} \tilde{V}^{2}+\tilde{\theta}_{1}^{\prime}\left[\tilde{V}\left[\Psi_{1}(x, u)-B_{1}(\alpha) \Phi\right]+\Gamma_{1}^{-1} \dot{\hat{\theta}}_{1}\right] \\
& =-k_{1} \tilde{V}^{2}
\end{aligned}
$$

To ensure non-singularity of the control law (7) over the envelope of feasible flight conditions it suffices to constrain the estimates $\hat{\theta}_{1}(t)$ to evolve within a suitable compact convex set $\Theta_{1} \subset \mathbb{R}^{16}$ constructed by allowing each parameter $\mathcal{P}_{i}$ that enters $\theta_{1}$ to vary within the set $\mathbb{P}_{\mathcal{P}_{i}}\left(\Xi_{\mathcal{P}}\right)$. This can be easily accomplished by resorting to smooth parameter projection methods in the definition of the update law [10].

\section{B. Adaptive Controller for the $(\tilde{h}, \tilde{\gamma})$-Subsystem}

Given a desired reference profile $h_{\text {ref }}(t)$ for the vehicle altitude, the corresponding tracking error, defined as $\tilde{h}(t)=$ $h(t)-h_{\text {ref }}(t)$, satisfies $\dot{\tilde{h}}=V_{\text {ref }} \gamma-\dot{h}_{\text {ref }}+\tilde{V} \sin \gamma$, since for

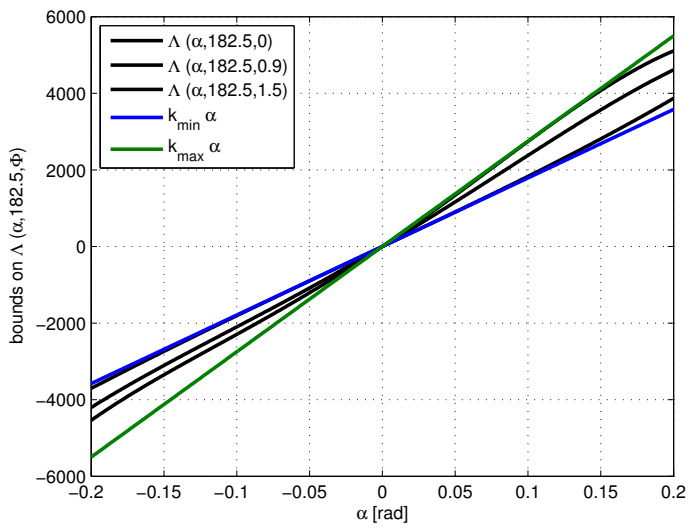

Fig. 1. Sector Boundedness Property of the Function $\Lambda\left(\alpha, \bar{q}_{c}, \Phi\right)$.

small values of the flight path angle $\sin \gamma \approx \gamma$. Choosing the desired trajectory for the flight-path angle as

$$
\gamma_{\mathrm{d}}=-\varepsilon \tilde{h}+\frac{1}{V_{\mathrm{ref}}} \dot{h}_{\mathrm{ref}}
$$

where $\varepsilon>0$ is a gain parameter, the corresponding dynamics for the altitude tracking error reads as

$$
\dot{\tilde{h}}=-\varepsilon V_{\text {ref }} \tilde{h}+V_{\text {ref }} \tilde{\gamma}+\tilde{V} \sin \gamma
$$

whereas the time derivative of $\gamma_{d}$ is given by

$\dot{\gamma}_{\mathrm{d}}=\varepsilon^{2} V_{\text {ref }} \tilde{h}-\varepsilon V_{\text {ref }} \tilde{\gamma}-\varepsilon \tilde{V} \sin \gamma-\frac{\dot{V}_{\text {ref }}}{V_{\text {ref }}^{2}} \dot{h}_{\text {ref }}+\frac{1}{V_{\text {ref }}} \ddot{h}_{\text {ref }}$.

The dynamics of the tracking error for the flight-path angle $\tilde{\gamma}=\gamma-\gamma_{\mathrm{d}}$ is written in the form

$$
\begin{aligned}
& \dot{\tilde{\gamma}}=\frac{1}{m V}\left[\bar{q} S C_{L}^{\alpha} \alpha+T(\alpha, \Phi) \sin \alpha+m g\left[[1-\cos \tilde{\gamma}] \cos \gamma_{\mathrm{d}}\right.\right. \\
& \left.\quad+\sin \tilde{\gamma} \sin \gamma_{\mathrm{d}}\right]-m g \cos \gamma_{\mathrm{d}}+\bar{q} S\left[C_{L}^{\delta_{e}} \delta_{e}+C_{L}^{\delta_{c}} \delta_{c}+C_{L}^{0}\right] \\
& \left.+\varepsilon m V V_{\text {ref }} \tilde{\gamma}-\varepsilon^{2} m V V_{\text {ref }} \tilde{h}+\varepsilon m V \tilde{V} \sin \gamma+d(\text { ref })\right]
\end{aligned}
$$

where $d($ ref $)=\frac{\dot{V}_{\text {ref }}(t)}{V_{\text {ref }}^{2}(t)} m V(t) \dot{h}_{\text {ref }}(t)-\frac{m V(t)}{V_{\text {ref }}(t)} \ddot{h}_{\text {ref }}(t)$.

Property 3.2: The function $\Lambda(\alpha, \bar{q}, \Phi):=\bar{q} S C_{L}^{\alpha} \alpha+$ $T(\alpha, \Phi) \sin \alpha$ satisfies a sector boundedness property, in the sense that there exist two functions $\underline{\Lambda}(\bar{q}, \Phi): \mathbb{R}^{2} \rightarrow$ $\mathbb{R}_{\geq 0}$ and $\bar{\Lambda}(\bar{q}, \Phi): \mathbb{R}^{2} \rightarrow \mathbb{R}_{\geq 0}$ such that $\underline{\Lambda}(\bar{q}, \Phi)|\alpha| \leq$ $|\bar{\Lambda}(\alpha, \bar{q}, \Phi)| \leq \bar{\Lambda}(\bar{q}, \Phi)|\alpha|$ for all admissible values of $\bar{q}$ and $\Phi$.

For the feasible range of variation of the velocity $V$ and the altitude $h$ reported in Table I, the dynamic pressure $\bar{q}$ satisfies: $182.5 \mathrm{psf} \leq \bar{q} \leq 4000 \mathrm{psf}$. To show that Property 3.2 holds, let us first notice that $\Lambda(0, \bar{q}, \Phi)=0$. Graphically, it is possible to see that for for any fixed $\bar{q}_{c} \in[182.5,4000]$, and for $\Phi$ and $\alpha$ inside their ranges, the functions $\Lambda\left(\alpha, \bar{q}_{c}, \Phi\right)$ can be bounded by two straight lines of appropriate slope. In particular, Fig. 1 shows that for $\bar{q}_{c}=182.5$ there exist two positive constants $k_{\min }$ and $k_{\max }$ such that $k_{\min } \cdot|\alpha| \leq|\Lambda(\alpha, 182.5, \Phi)| \leq k_{\max } \cdot|\alpha|$ 
for any feasible $\alpha$ and $\Phi$. It is possible to show that for any feasible $\bar{q}$ such $k_{\min }$ and $k_{\max }$ exist, and $k_{\min }(\bar{q})$ and $k_{\max }(\bar{q})$ can be approximated by linear functions of $\bar{q}$. As a consequence, considering the expression of $\bar{q}$, the range of variation of $h, \Phi$ and $\bar{q}$ we can conclude that

$$
\Lambda(\alpha, \bar{q}(V, h), \Phi):=K(t) \cdot V^{2} \alpha
$$

where $K(t)$ is a time-varying coefficient that satisfies $k_{m} \leq$ $K(t) \leq k_{M}$ for two positive constants $k_{m}$ and $k_{M}$.

The desired angle of attack is selected as $\alpha_{d}=\alpha^{*}-\tilde{\gamma}$ where $\alpha^{*}$ can be set to arbitrary values in the envelope of the feasible flight conditions. Introducing $\tilde{\alpha}=\alpha-\alpha_{d}$, the angle of attack satisfies $\alpha=\alpha^{*}-\tilde{\gamma}+\tilde{\alpha}$ Using (10), it follows that

$$
\begin{aligned}
& \bar{q} S C_{L}^{\alpha} \alpha+T(\alpha, \Phi) \sin \alpha= \\
& \quad=\bar{q} S C_{L}^{\alpha}\left[\alpha^{*}-\tilde{\gamma}+\tilde{\alpha}\right]+T\left(\alpha^{*}-\tilde{\gamma}+\tilde{\alpha}, \Phi\right) \sin \left(\alpha^{*}-\tilde{\gamma}+\tilde{\alpha}\right) \\
& \quad:=\bar{q} c_{1} \alpha^{*}+c_{2}\left(\alpha^{*}\right) \Phi+c_{3}\left(\alpha^{*}\right)+K(t) \cdot V^{2}[-\tilde{\gamma}+\tilde{\alpha}]
\end{aligned}
$$

where $c_{1}$ is a constant, and $c_{2}(\cdot)$ and $c_{3}(\cdot)$ are continuous functions. Introducing the vectors of uncertain parameters $\theta_{2}$ and the regressor $\Psi_{2}$ defined as

$$
\begin{aligned}
& \theta_{2}=\left[\frac{C_{L}^{\delta_{e}}}{C_{L}^{\delta_{c}}}, \frac{C_{L}^{0}}{C_{L}^{\delta_{c}}}+\frac{c_{1} \alpha^{*}}{S C_{L}^{\delta_{c}}}, \frac{c_{2}\left(\alpha^{*}\right)}{S C_{L}^{\delta_{c}}}, \frac{c_{3}\left(\alpha^{*}\right)}{S C_{L}^{\delta_{c}}}, \frac{m}{S C_{L}^{\delta_{c}}}\right]^{\prime} \\
& \Psi_{2}=\left[-\delta_{e},-1,-\frac{\Phi}{\bar{q}},-\frac{1}{\bar{q}}, \frac{1}{\bar{q}}\left[g \cos \gamma_{\mathrm{d}}-\frac{\dot{V}_{\mathrm{ref}}}{V_{\mathrm{ref}}^{2}} V \dot{h}_{\mathrm{ref}}+\frac{V \ddot{h}_{\mathrm{ref}}}{V_{\mathrm{ref}}}\right]\right]^{\prime}
\end{aligned}
$$

the $\tilde{\gamma}$ dynamics read as

$$
\begin{aligned}
\dot{\tilde{\gamma}}= & \frac{1}{m V}\left[K(t) \cdot V^{2}(-\tilde{\gamma}+\tilde{\alpha})+m g\left[(1-\cos \tilde{\gamma}) \cos \gamma_{\mathrm{d}}\right.\right. \\
& \left.+\sin \tilde{\gamma} \sin \gamma_{\mathrm{d}}\right]+\bar{q} S C_{L}^{\delta_{c}}\left(\delta_{c}-\Psi_{2}^{\prime} \theta_{2}\right)+\varepsilon m V V_{\text {ref }} \tilde{\gamma} \\
& \left.-\varepsilon^{2} m V V_{\text {ref }} \tilde{h}+\varepsilon m V \tilde{V} \sin \gamma\right] .
\end{aligned}
$$

Using (5), it follows that

$$
\left|[1-\cos \tilde{\gamma}] \cos \gamma_{\mathrm{d}}+\sin \tilde{\gamma} \sin \gamma_{\mathrm{d}}\right| \leq \frac{\tilde{\gamma}^{2}}{2}+|\sin \tilde{\gamma}|\left|\sin \gamma_{\mathrm{d}}\right|
$$

and therefore

$$
\begin{gathered}
-K(t) \cdot V^{2} \tilde{\gamma}+m g\left[[1-\cos \tilde{\gamma}] \cos \gamma_{\mathrm{d}}+\sin \tilde{\gamma} \sin \gamma_{\mathrm{d}}\right] \\
:=-K_{S}(t) \cdot V^{2} \tilde{\gamma}
\end{gathered}
$$

where $K_{S}(t)$ is a time-varying coefficient such that $K_{S}(t)>$ $k_{s}>0$. Moreover, for all $\mathcal{P} \in \Xi_{\mathcal{P}}$

$$
\bar{q} S C_{L}^{\delta_{c}}:=K_{C}(t) \cdot V^{2} \geq k_{c} \cdot V^{2}
$$

where $K_{C}(t)$ is a time-varying coefficient and $k_{c}$ is a positive constant. Let us introduce the parameter estimate vector $\hat{\theta}_{2}$, the parameter estimate error $\tilde{\theta}_{2}=\hat{\theta}_{2}-\theta_{2}$ and the Lyapunov function candidate

$$
W_{2}\left(\tilde{h}, \tilde{\gamma}, \tilde{\theta}_{2}\right)=\frac{\varepsilon_{0}}{2} \tilde{h}^{2}+\frac{m}{2} \tilde{\gamma}^{2}+\frac{S C_{L}^{\delta_{c}}}{2} \tilde{\theta}_{2}^{\prime} \Gamma_{2}^{-1} \tilde{\theta}_{2},
$$

where $\Gamma_{2}$ is a symmetric positive definite matrix and the scaling parameter $\varepsilon_{0}>0$ needs to be chosen in such a way that, for all admissible values $m$ of the mass of the vehicle, condition $\mathcal{C} .1$ is satisfied for some $c>0$.
Proposition 3.3: Choosing the canard deflection input as

$$
\delta_{c}=\Psi_{2}^{\prime} \hat{\theta}_{2}-k_{2} \tilde{\gamma}
$$

where $k_{2}>0$ is a gain parameter, and the update law for the parameter estimate $\hat{\theta}_{2}$ as

$$
\dot{\hat{\theta}}_{2}=-\Gamma_{2} \frac{\bar{q} \Psi_{2} \tilde{\gamma}}{V},
$$

there exists a value $k_{2}^{*}$ such that for all $k_{2}>k_{2}^{*}$ the Lie derivative of $W_{2}\left(\tilde{h}, \tilde{\gamma}, \hat{\theta}_{2}\right)$ along the trajectories of the system is negative semidefinite when $\tilde{\alpha}=\tilde{V}=0$.

Proof: The Lie derivative of $W_{2}\left(\tilde{h}, \tilde{\gamma}, \tilde{\theta}_{2}\right)$ along the trajectories of system is given by:

$$
\begin{aligned}
\dot{W}_{2}= & -\varepsilon \varepsilon_{0} V_{\text {ref }} \tilde{h}^{2}+\left[\varepsilon_{0} V_{\text {ref }}-\varepsilon^{2} m V_{\text {ref }}\right] \tilde{\gamma} \tilde{h} \\
& -\left[K_{S}(t) V+k_{2} K_{C}(t) V-\varepsilon m V_{\text {ref }}\right] \tilde{\gamma}^{2} \\
& +K(t) V \tilde{\alpha} \tilde{\gamma}+\varepsilon_{0} \tilde{V} \tilde{h} \sin \gamma+\varepsilon m \tilde{V} \tilde{\gamma} \sin \gamma \\
& +\frac{\bar{q} S C_{L}^{\delta_{c}}}{V} \tilde{\theta}_{2}^{\prime}\left[\Psi_{2} \tilde{\gamma}+\frac{V}{\bar{q}} \Gamma_{2}^{-1} \dot{\hat{\theta}}_{2}\right] .
\end{aligned}
$$

Using the bounds previously derived it follows that

$$
\begin{aligned}
\dot{W}_{2} \leq & -V_{\text {ref }}[|\tilde{h}|,|\tilde{\gamma}|] Q[|\tilde{h}|,|\tilde{\gamma}|]^{\prime} \\
& +K(t) V \tilde{\alpha} \tilde{\gamma}+\varepsilon_{0} \tilde{V} \tilde{h} \sin \gamma+\varepsilon m \tilde{V} \tilde{\gamma} \sin \gamma
\end{aligned}
$$

where

$$
Q:=\left[\begin{array}{cc}
\varepsilon \varepsilon_{0} & \frac{\varepsilon^{2} m-\varepsilon_{0}}{2} \\
\frac{\varepsilon^{2} m-\varepsilon_{0}}{2} & k_{s}+k_{c} k_{2}-\varepsilon m
\end{array}\right] .
$$

Since for any value of $\varepsilon$ and $\varepsilon_{0}$ there $\exists k_{2}^{*}>0$ such that

$$
k_{s}+k_{c} k_{2}^{*} \geq \varepsilon m+\frac{1}{4 \varepsilon \varepsilon_{0}}\left(\varepsilon^{2} m-\varepsilon_{0}\right)^{2},
$$

using Sylvester's Criterion it follows that for any $k_{2}>k_{2}^{*}$, the matrix $Q$ is positive definite. Let $\lambda_{Q}$ be the smallest eigenvalue of $Q$; by the positive definiteness property of $Q$, it follows that $\lambda_{Q}>0$, and $\dot{W}_{2} \leq-V_{\text {ref }} \lambda_{Q}\left[\tilde{h}^{2}+\tilde{\gamma}^{2}\right]$ for $\tilde{\alpha}=\tilde{V}=0$, .

\section{Robust Controller for the $(\tilde{\alpha}, Q)$-Subsystem}

Using (1), the $(\tilde{\alpha}, Q)$ subsystem is written as

$$
\begin{aligned}
\dot{\tilde{\alpha}} & =Q-\dot{\gamma}_{\mathrm{d}} \\
I_{y y} \dot{Q} & =M .
\end{aligned}
$$

To let $\alpha(t)$ remain close to the desired trim value $\alpha^{*}$, we augment the system with an integrator $\xi$ whose dynamics are given by $\dot{\xi}=\tilde{\alpha}$. Introducing the change of coordinates $\chi_{1}=\tilde{\alpha}+\mu \xi, \chi_{2}=\mu \tilde{\alpha}+Q-\dot{\gamma}_{\mathrm{d}}$, the $\left(\xi, \chi_{1}, \chi_{2}\right)$ subsystem is given by

$$
\begin{aligned}
\dot{\xi} & =-\mu \xi+\chi_{1} \\
\dot{\chi}_{1} & =\chi_{2} \\
\dot{\chi}_{2} & =\mu \chi_{2}-\mu^{2} \chi_{1}+\mu^{3} \xi+\frac{M}{I_{y y}}-\ddot{\gamma}_{\mathrm{d}} .
\end{aligned}
$$

The desired value for the pitching moment is selected as

$$
M_{d}=I_{y y_{\circ}}\left[\left[-\mu-k_{3} a_{1}\right] \chi_{2}+\left[\mu^{2}-k_{3}^{2} a_{0}\right] \chi_{1}-\mu^{3} \xi\right]
$$


where $k_{3}, I_{y y_{o}}>0$ are gain parameters and $a_{1}$ and $a_{0}$ are positive constant. Let $\bar{a}_{1}:=\left(I_{y y_{o}} / I_{y y}\right) a_{1}$ and $\bar{a}_{0}:=$ $\left(I_{y y_{o}} / I_{y y}\right) a_{0}$; then defining $\tilde{I}_{y y}:=1-\left(I_{y y_{o}} / I_{y y}\right)$, the $\chi_{2}$ dynamic equation reads as

$$
\begin{aligned}
\dot{\chi}_{2}=- & k_{3} \bar{a}_{1} \chi_{2}-k_{3}^{2} \bar{a}_{0} \chi_{1}+\frac{1}{I_{y y}}\left[\tilde { I } _ { y y } I _ { y y } \left[\mu \chi_{2}-\mu^{2} \chi_{1}\right.\right. \\
& \left.\left.+\mu^{3} \xi\right]+M-M_{d}-I_{y y} \ddot{\gamma}_{\mathrm{d}}\right] .
\end{aligned}
$$

Introducing the vector of uncertain parameters $\theta_{3}$, the regressor $\Psi_{3}$ and the input matrix $B_{3}$ respectively as

$$
\begin{gathered}
\theta_{3}=\left[z_{T} C_{T}^{\Phi \alpha^{3}}, z_{T} C_{T}^{\Phi \alpha^{2}}, z_{T} C_{T}^{\Phi \alpha}, z_{T} C_{T}^{\Phi}, z_{T} \bar{C}_{T}^{3},\right. \\
z_{T} \bar{C}_{T}^{2}, z_{T} \bar{C}_{T}^{1}, z_{T} \bar{C}_{T}^{0}, S \bar{c} C_{M}^{\alpha^{2}}, S \bar{c} C_{M}^{\alpha}, S \bar{c} C_{M}^{\delta_{e}}, \\
\left.S \bar{c} C_{M}^{\delta_{c}}, S \bar{c} C_{M}^{0}, \tilde{I}_{y y} I_{y y}, I_{y y}\right]^{\prime} \\
\Psi_{3}(x, u)=\left[-\alpha^{3} \Phi,-\alpha^{2} \Phi,-\alpha \Phi,-\Phi,-\alpha^{3},-\alpha^{2},-\alpha,-1,\right. \\
\left.-\bar{q} \alpha^{2},-\bar{q} \alpha, 0,-\bar{q} \delta_{c},-\bar{q},-\mu \chi_{2}+\mu^{2} \chi_{1}-\mu^{3} \xi, \ddot{\gamma}_{\mathrm{d}}\right]^{\prime} \\
B_{3}(\bar{q})=[0,0,0,0,0,0,0,0,0,0, \bar{q}, 0,0,0,0]^{\prime},
\end{gathered}
$$

the $\left(\xi, \chi_{1}, \chi_{2}\right)$ dynamics can be written in the following form

$$
\begin{aligned}
\dot{\xi}= & -\mu \xi+\chi_{1} \\
\dot{\chi}_{1}= & \chi_{2} \\
\dot{\chi}_{2}= & -k_{3} \bar{a}_{1} \chi_{2}-k_{3}^{2} \bar{a}_{0} \chi_{1} \\
& +\frac{1}{I_{y y}}\left[\theta_{3}^{\prime} B_{3}(\bar{q}) \delta_{e}-\Psi_{3}^{\prime}(x, u) \theta_{3}-M_{d}\right] .
\end{aligned}
$$

The change of coordinates $\zeta_{1}=\chi_{1}, \zeta_{2}=\chi_{2} / k_{3}$ transforms (13) into

$$
\begin{aligned}
& \dot{\xi}=-\mu \xi+\zeta_{1} \\
& \dot{\zeta}=-k_{3} F \zeta+\frac{G}{I_{y y}}\left[\theta_{3}^{\prime} B_{3}(\bar{q}) \delta_{e}-\Psi_{3}^{\prime}(x, u) \theta_{3}-M_{d}\right],
\end{aligned}
$$

where

$$
\zeta=\left[\begin{array}{l}
\zeta_{1} \\
\zeta_{2}
\end{array}\right], \quad F=\left[\begin{array}{cc}
0 & 1 \\
-\bar{a}_{0} & -\bar{a}_{1}
\end{array}\right] \text { and } G=\left[\begin{array}{c}
0 \\
1 \\
\hline k_{3}
\end{array}\right]
$$

Since $p(\lambda)=\lambda^{2}+\bar{a}_{1} \lambda+\bar{a}_{0}$ is a Hurwitz polynomial, there exists a symmetric and positive definite matrix $P$ satisfying $F^{\prime} P+P F \leq-I$ for all $I_{y y} \in \mathbb{P}_{I_{y y}}\left(\Xi_{\mathcal{P}}\right)$. Introduce the parameter estimate vector $\hat{\theta}_{3}$, the parameter estimate error $\tilde{\theta}_{3}=\hat{\theta}_{3}-\theta_{3}$ and the Lyapunov function candidate

$W=W_{1}\left(\tilde{V}, \tilde{\theta}_{1}\right)+W_{2}\left(\tilde{h}, \tilde{\gamma}, \tilde{\theta}_{2}\right)+\frac{1}{2} \xi^{2}+\zeta^{\prime} P \zeta+\frac{1}{I_{y y}} \tilde{\theta}_{3}^{\prime} \Gamma_{3}^{-1} \tilde{\theta}_{3}$

where $\Gamma_{3}$ is a symmetric positive definite matrix.

Proposition 3.4: Choosing the elevator deflection input as

$$
\delta_{e}=\frac{1}{\hat{\theta}_{3}^{\prime} B_{3}}\left[\Psi_{3}^{\prime} \hat{\theta}_{3}+M_{d}\right]
$$

and the update law for the parameter estimate $\hat{\theta}_{3}$ as

$$
\dot{\hat{\theta}}_{3}=\Gamma_{3}\left[B_{3} \delta_{e}-\Psi_{3}\right] G^{\prime} P \zeta,
$$

it follows that there exist $k_{1}^{*}, k_{2}^{*}, k_{3}^{*}$ and $\mu^{*}$ such that for all $k_{1}>k_{1}^{*}, k_{2}>k_{2}^{*}, k_{3}>k_{3}^{*}$ and $\mu<\mu^{*}$ the derivative of $W$ along trajectories of the closed-loop system is negative semi-definite.
TABLE II

\begin{tabular}{cl|ll}
\multicolumn{4}{c}{ DESIGN PARAMETERS AND GAINS VALUE } \\
Gain & Value & Parameter & Value \\
\hline$k_{1}$ & 200 & $\mu$ & 0.75 \\
$k_{2}$ & 200 & $\epsilon$ & $1 \mathrm{e}-004$ \\
$k_{3}$ & 40 & $a_{0}$ & 0.5 \\
$\Gamma_{1}$ & $0.1 \times I_{16 \times 16}$ & $a_{1}$ & 1.5 \\
$\Gamma_{2}$ & $0.1 \times I_{5 \times 5}$ & $I_{y y}$ & 86722 \\
$\Gamma_{3}$ & $0.1 \times I_{13 \times 13}$ & $\alpha^{*}$ & $0.035[\mathrm{rad}]$
\end{tabular}

Proof: Using Prepositions 3.1 and 3.3, the Lie derivative of $W$ along the trajectories of the system satisfies

$$
\begin{aligned}
\dot{W} \leq & -k_{1} \tilde{V}^{2}-V_{\text {ref }}(t) \lambda_{Q}\left[\tilde{h}^{2}+\tilde{\gamma}^{2}\right]-\mu \xi^{2}+\xi \zeta_{1}-k_{3}\left[\zeta_{1}^{2}+\zeta_{2}^{2}\right] \\
& +K(t) V \tilde{\gamma}\left[\zeta_{1}-\mu \xi\right]+\varepsilon_{0} \tilde{V} \tilde{h} \sin \gamma(t)+\varepsilon m \tilde{V} \tilde{\gamma} \sin \gamma(t) \\
& =-\left[\tilde{V}, \tilde{h}, \tilde{\gamma}, \xi, \zeta_{1}, \zeta_{2}\right]^{\prime} R(t)\left[\tilde{V}, \tilde{h}, \tilde{\gamma}, \xi, \zeta_{1}, \zeta_{2}\right]
\end{aligned}
$$

where $R(t)$ is a time-varying matrix whose expression is not reported here for reasons of space limitation. Let $\Delta_{i}$ denote the determinant associated with $i$-th order upper-left submatrix of $R(t)$. Let $k_{2}^{*}$ be defined by condition (11), then any choice of $k_{2}>k_{2}^{*}$ and $k_{1}$ such that

$$
k_{1}>k_{1}^{*}=\frac{\varepsilon_{0}^{2}+\varepsilon^{2} m^{2}}{4 \lambda_{Q} \min _{t>0} V_{\text {ref }}(t)}
$$

guarantees that $\Delta_{1}, \Delta_{2}$ and $\Delta_{3}$ are strictly positive. Moreover, any choice of $\mu$ that satisfies

$$
\mu<\mu^{*}=\frac{4 \Delta_{3}}{\Delta_{2}} \frac{1}{K_{M}^{2} \max _{t>0} V^{2}(t)}
$$

renders $\Delta_{4}$ strictly positive as well. Finally, any choice of $k_{3}$ compatible with $k_{3}>k_{3}^{*}=\Delta_{3} / 4 \Delta_{4}$ guarantees that also $\Delta_{5}$ and $\Delta_{6}$ are strictly positive. The result follows by applying Sylvester's criterion.

Standard arguments [11] imply that all trajectories of the closed-loop system are bounded, and that the tracking errors $\tilde{V}(t), \tilde{h}(t)$ and $\tilde{\gamma}(t)$ are regulated to zero asymptotically.

\section{Simulations}

To validate the controller derived in the previous section, simulations have been performed on the HFM model implemented in SIMULINK ${ }^{\circledR}$. As a representative case study, the vehicle is initially trimmed at $h=85000 \mathrm{ft}$ and $V=$ $7846.36 \mathrm{ft} / \mathrm{s}$; the reference trajectory $h_{\text {ref }}(t)$ is generated to let the vehicle climb $25000 \mathrm{ft}$ in about $300 s$, and $V_{\text {ref }}(t)$ is generated to increase the vehicle velocity of about $2650 \mathrm{ft} / \mathrm{s}$ in the same time interval. The reference commands have been generated by filtering step increments in velocity and altitude while the tracking reference for the flight path angle is generated using (8). The initial conditions of the plant parameter estimates have been randomly selected within $40 \%$ of their nominal values. The controller gains are reported in Table II. Fig. 2-3 show that the tracking performance in closed-loop for the velocity and altitude are excellent in spite of parameter uncertainty and the presence of the flexible dynamics, neglected in the controller design. The flexible states remain well-behaved while the angle of attack remains close to the desired trim value, as shown in Fig.4. 

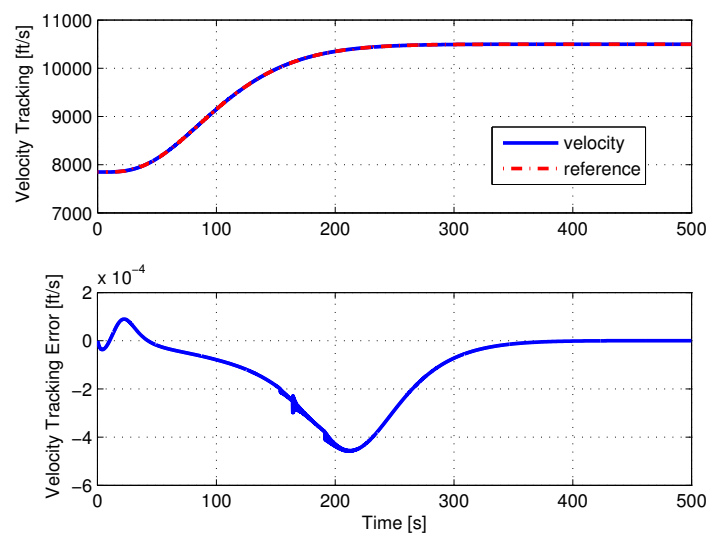

Fig. 2. Velocity Tracking and Tracking Error
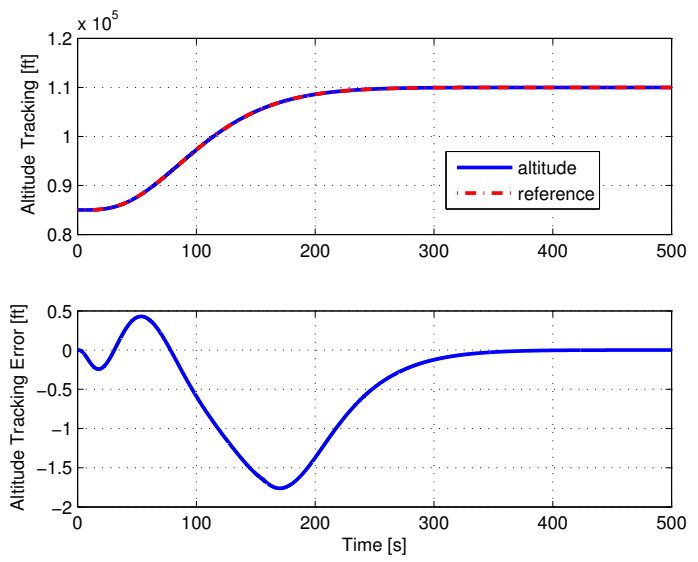

Fig. 3. Altitude Tracking and Tracking Error

Finally, Fig. 5 shows that the control effort remains within the feasible range.

\section{CONCLusions}

In this paper we have presented an improved robust/adaptive nonlinear controller design for the longitudinal motion of an air-breathing hypersonic vehicle. The controller does not depend explicitly on the model coefficients and therefore provides robustness with respect to model uncertainties. With respect to our previous work, the stability analysis has been simplified, and a desired trim value for the angle of attack can now be imposed. To further improve the performance of the controller, current work is focused on including the presence of flexible effects directly at the design level.

\section{REFERENCES}

[1] C. Tournes, D. B. Landrum, Y. Shtessel, and C. W. Hawk, "Ramjetpowered reusable launch vehicle control by sliding modes," AIAA Journal of Guidance, Control, and Dynamics, vol. 21, no. 3, pp. 40915, 1998.

[2] D. B. Doman and A. D. Ngo, "Dynamic inversion-based adaptive/reconfigurable control of the X-33 on ascent," AIAA Journal of Guidance, Control, and Dynamics, vol. 25, no. 2, pp. 275-84, 2002.
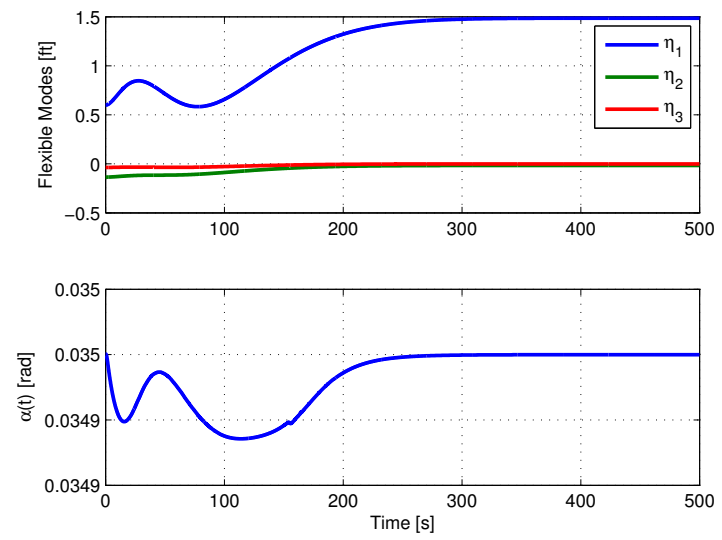

Fig. 4. Flexible States and Angle of Attack
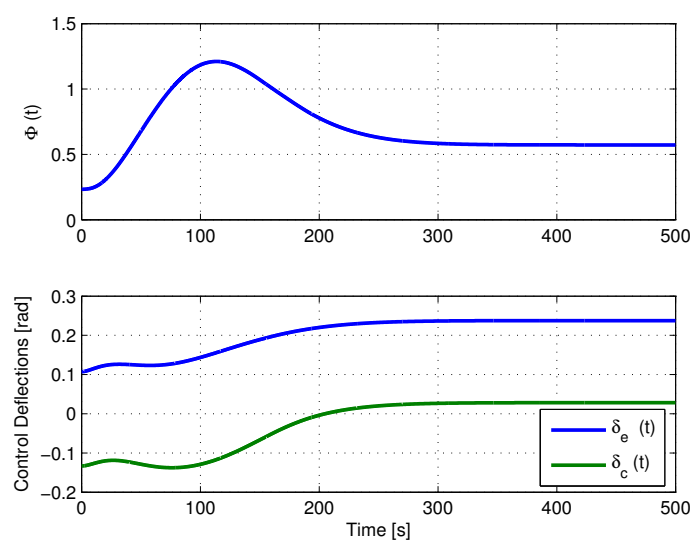

Fig. 5. Control Inputs

[3] Q. Wang and R. Stengel, "Robust nonlinear control of a hypersonic aircraft," AIAA Journal of Guidance, Control, and Dynamics, vol. 23, no. 4, pp. 577-85, 2000.

[4] H. Xu, M. Mirmirani, and P. Ioannou, "Adaptive sliding mode control design for a hypersonic flight vehicle," AIAA Journal of Guidance, Control, and Dynamics, vol. 27, no. 5, pp. 829-38, 2004.

[5] J. T. Parker, A. Serrani, S. Yurkovich, M. A. Bolender, and D. B. Doman, "Control-oriented modeling of an air-breathing hypersonic vehicle," Journal of Guidance, Control, and Dynamics, vol. 30, no. 3, pp. 856-869, 2007.

[6] M. A. Bolender and D. B. Doman, "A nonlinear longitudinal dynamical model of an air-breathing hypersonic vehicle," Journal of Spacecraft and Rockets, vol. 44, no. 2, pp. 374-387, 2007.

[7] L. Fiorentini, A. Serrani, M. Bolender, and D. Doman, "Nonlinear robust/adaptive controller design for an air-breathing hypersonic vehicle model," in AIAA Guidance, Navigation and Control Conference and Exhibit, Hilton Head, SC, 2007, AIAA Paper 2007-6329.

[8] T. Williams, M. A. Bolender, D. B. Doman, and O. Morataya, "An aerothermal flexible mode analysis of a hypersonic vehicle," in AIAA Atmospheric Flight Mechanics Conference and Exhibit, Keystone, CO, Aug. 2006, AIAA Paper 2006-6647.

[9] J. Schierman, D. Ward, J. Hull, N. Gandhi, M. Oppenheimer, and D. Doman, "Integrated adaptive guidance and control for re-entry vehicles with flight-test results," AIAA Journal of Guidance, Control, and Dynamics, vol. 27, no. 6, pp. 975-88, 2004.

[10] H. K. Khalil, "Adaptive output feedback control of nonlinear systems represented by input-output models," IEEE Transactions on Automatic Control, vol. 41, no. 2, pp. 177-88, 1996.

[11] H. Khalil, Nonlinear Systems, 3rd ed. Upper Saddle River, NJ: Prentice Hall, 2002. 